\title{
El servicio público de abastecimiento de agua potable a varios municipios por una misma empresa municipal
}

\author{
M. ${ }^{a}$ Asunción Torres López \\ Profesora Titular de Derecho Administrativo \\ Universidad de Granada
}

\begin{abstract}
SUMARIO: I. INTRODUCCIÓN. II. EL ABASTECIMIENTO DOMICILIARIO DE AGUA A VARIAS POBLACIONES: EL ARTÍCULO 89 DE LA LEY DE AGUAS DE 2001. III. LA DISTRIBUCIÓN DE COMPETENCIAS EN MATERIA DE AGUAS ENTRE LAS DISTINTAS ADMINISTRACIONES PÚBLICAS DE BASE TERRITORIAL. 1. La distribución de competencias entre el Estado y las Comunidades Autónomas en materia de aguas. 1.1. La distribución de competencias en la CE del 78. 1.2. Legislación de aguas y la confirmación de la distribución de competencias en ella contenida por el TC. 1.3. Las cuencas hidrográficas como criterio esencial de distribución de competencias. Concepto y funcionalidad de la «cuenca hidrográfica». 1.4. La legislación sectorial de las CC.AA. y su interrelación con la competencia estatal en materia de aguas. 2. Competencias de la Administración local en materia de recursos hidráulicos. 2.1. Introducción. 2.2. El servicio de abastecimiento de agua a poblaciones. 2.3. El abastecimiento a poblaciones como servicio público. III. FORMAS DE GESTIÓN DEL SERVICIO PÚBLICO DE ABASTECIMIENTO DE AGUA POTABLE A VARIOS MUNICIPIOS. 1. Introducción. 2. Alternativas de gestión para la prestación del servicio público de abastecimiento a varias poblaciones: el artículo 89 LA. 2.1. Constitución de los municipios afectados en Mancomunidad, Consorcio o Entidades semejantes. 2.2. El servicio de abastecimiento de agua potable a varios municipios prestado por una misma empresa concesionaria. 2.2.1. Creación por todos los municipios afectados de una nueva empresa de aguas. 2.2.2. Prestación del servicio por la empresa de aguas que presta dicho servicio en el municipio económicamente más importante: la conversión de un servicio público local de carácter esencial en una actividad competitiva.
\end{abstract}

\section{INTRODUCCIÓN}

En el presente artículo se expone la problemática de la gestión del servicio de abastecimiento de aguas a varios municipios por una misma empresa municipal, aunque participada tan sólo por uno de los municipios. La utilización de la figura del convenio interadministrativo sobre la base de la potestad de autoorganización que corresponde a las Administraciones Públicas y de la cooperación administrativa para la prestación de los servicios públicos, ha servido en la práctica para proceder a una verdadera «adjudicación directa» de un contrato de gestión de servicios públicos, eludiendo ilícitamente los principios de publicidad y concurrencia que exigen las normas de la contratación administrativa. 
Pretende ser ésta una pequeña aportación al Derecho de Aguas que de manera tan profunda, práctica y constante ha desarrollado el Profesor Sebastián MARTíN-RetortiLlo BAQUer, de quien hemos aprendido en nuestro estudio continuado y cuya obra florecerá siempre como un referente en nuestras enseñanzas.

\section{EL ABASTECIMIENTO DOMICILIARIO DE AGUA A VARIAS POBLACIONES: EL ARTÍCULO 89 DE LA LEY DE AGUAS DE 2001}

La vigente Ley de Aguas (R.D.Legislativo 1/2001, de 20 de julio), condiciona el otorgamiento de concesiones para abastecimiento a varias poblaciones, en su artículo 89.1, a que:

1. Las Corporaciones Locales estén constituidas a estos efectos en Mancomunidades, Consorcios u otras entidades semejantes, de acuerdo con la legislación por la que se rijan; o bien a que

2. Todas ellas reciban el agua a través de una misma empresa concesionaria.

Esta segunda alternativa que permite la Ley elude o evita el primer supuesto, esto es, la formación obligada de «mancomunidades, consorcios u otras entidades semejantes».

El estudio de ambas alternativas que permite la Ley nos lleva, en primer lugar, a definir las competencias de cada una de las Administraciones implicadas: la Administración local, al tratarse el abastecimiento de un servicio público competencia de esta Administración; la Administración estatal a través de la Confederación Hidrógráfica que sea competente para otorgar las concesiones para utilización de aguas públicas; y la Comunidad Autónoma, cuyo grado de intervención será mayor o menor en función del desarrollo normativo llevado a cabo que, en ningún caso, podrá menoscabar las competencias locales, aunque sí que puede condicionarlas.

No pretendemos realizar un estudio de la distribución de competencias en materia de aguas, aspecto ampliamente tratado por la doctrina en numerosos estudios ${ }^{1}$; sin embargo, debemos destacar, siquiera brevemente, los

\footnotetext{
${ }^{1}$ Véase S. Martín-Retortillo Baquer, Aguas públicas y obras hidráulicas, Madrid, 1996; Derecho de Aguas, Civitas, Madrid, 1997; «Acotaciones sobre el nuevo Derecho de Aguas», REDA, núm. 101 (1999); A. EmBiD Irujo (Dir.) La reforma de la Ley de Aguas (Ley 46/1999, de 13 de Diciembre), Civitas, Madrid, 2000; «Las competencias sobre las aguas continentales. Plantea-
} 
títulos de intervención de cada una de estas Administraciones Públicas, siendo conscientes de la necesaria colaboración que ha de prestarse entre las Administraciones públicas de base territorial para una gestión integral del agua potable en el entorno de varios municipios.

\section{LA DISTRIBUCIÓN DE COMPETENCIAS EN MATERIA DE AGUAS ENTRE LAS DISTINTAS ADMINISTRACIONES PÚBLICAS DE BASE TERRITORIAL}

\section{La distribución de competencias entre el Estado y las Comunidades Autónomas en materia de aguas}

\subsection{La distribución de competencias en la CE del 78}

La CE, aunque de forma ambigua y confusa, delimita en sus artículos 148 y 149 las competencias que tanto al Estado como a las CC.AA. les pertenecen. No es, sin embargo, una delimitación técnicamente bien realizada; por el contrario, se trata de una distribución de competencias incompleta que casi siempre ha tenido que precisarse a través de los fallos pronunciados por el Tribunal Constitucional.

En el caso de la Administración Local, que es la que más nos interesa a los efectos de este artículo, como Administración de base territorial (municipios y provincias fundamentalmente) y dotada constitucionalmente de autonomía para la gestión de sus intereses, la atribución constitucional de competencias utiliza un mecanismo distinto al de las otras dos Administra-

\footnotetext{
miento normativo y realidad jurídica», Legislación del agua en las Comunidades Autónomas, Tecnos, Madrid, 1993; A. Fanlo Loras, «La articulación de las competencias de las Comunidades Autónomas en la gestión del agua», en AA.VV., Gestión del agua y medio ambiente (dir. EMBID Irujo), Civitas, Madrid, 1995; Las Confederaciones Hidrográficas y otras Administraciones Hidráulicas, Civitas, Madrid, 1996; A. Molina GimÉneZ, El servicio público de abastecimiento de agua en poblaciones. El contexto liberalizador, Tirant lo Blanch, Valencia, 2001; J.L. MeILÁn y J. Rodríguez-Arana, «Las competencias del Estado y de las Comunidades Autónomas en materia de aguas: especial referencia a los casos gallego y canario», Autonomies, núm. 11 (1990); P. MeNÉNDEZ, «Una interpretación renovada de la reserva de servicios esenciales a favor de las entidades locales, especial referencia al abastecimiento de aguas y suministro de electricidad», REDA, núm. 89 (1996); A. SÁNCHEZ BLANCO, «Distribución constitucional de competencias en materia de recursos naturales, aguas, minas y montes», Estudios sobre la Constitución Española. Libro Homenaje al Profesor Eduardo García de Enterría, Civitas, Tomo IV, Madrid, 1991. C. GonZÁlez-Antón Álvarez, El uso del agua por las Administraciones Locales, Cedecs, Barcelona, 2000; F. DelgaDo PiQueras, «La distribución de competencias Estado-Comunidades Autónomas en materia de aguas: la Sentencia del Tribunal Constitucional de 29 de noviembre de 1988», RAP, núm. 118 (1989); L. Morell Ocaña, El régimen local español, Civitas, Madrid, 1988; L. Ortega Álvarez, «Las competencias propias de las corporaciones locales», en MuÑoz MACHADo (dir.), Tratado de Derecho Municipal, Civitas, Madrid, 1988.
} 
ciones territoriales. Mientras que Estado y CC.AA. tienen potestad legislativa en sentido estricto $y$, por lo tanto, pueden dictar leyes en función de la atribución de competencias que tiene lugar en la propia CE (arts. 148 y 149 fundamentalmente), en el caso de la Administración local, la atribución de competencias se realiza mediante normas de rango legal emanadas de una u otra Administración. Es decir, el contenido competencial de los entes locales vendrá determinado por la legislación sectorial y consistirá en competencias propias, con distinto alcance, y en competencias delegadas.

Como hemos dicho, los dos preceptos que fundamentalmente llevan a cabo en nuestra CE la distribución de competencias entre Estado y CC.AA. son el 148 y 149. Los dos apartados que se refieren directamente a la materia de los recursos hidráulicos son el 149.1.22. ${ }^{\mathrm{a}}$ y el 148.1.10. ${ }^{\mathrm{a}}$. El artículo 149.1.22. ${ }^{\mathrm{a}} \mathrm{CE}$ atribuye al Estado competencia exclusiva sobre «la legislación, ordenación y concesión de recursos y aprovechamientos hidráulicos cuando las aguas discurran por más de una Comunidad Autónoma». Por su parte, el artículo 148.1.10. a permite a las CC.AA. asumir competencias sobre «los proyectos, construcción y explotación de los aprovechamientos hidráulicos, canales y regadíos de interés de la Comunidad Autónoma; las aguas minerales y termales» (es decir, competencias sobre obras hidráulicas y aguas minerales y termales).

El criterio de atribución de competencias en estos dos preceptos genéricos no es, sin embargo, homogéneo. Así, en el caso de las competencias autonómicas es el «interés autonómico» el determinante de la atribución competencial a éstas. Por el contrario, en el caso de la competencia estatal el criterio de atribución de competencias es un elemento puramente geográfico y relativo al bien de que se trata: «el agua que discurra por más de una Comunidad Autónoma».

Éstos serían los preceptos genéricos de atribución global de competencias en materia de aguas. Sin embargo, sobre las aguas, como objeto de regulación y gestión, concurre una pluralidad de títulos competenciales, tanto estatales como autonómicos. La razón de esta pluralidad competencial es que el agua es el soporte de multitud de actividades públicas y privadas (ordenación del territorio, medio ambiente, obras públicas, regadíos, vertidos, pesca fluvial, régimen energético, montes, etc). Interrelación e interferencia de determinados títulos competenciales con influencia en la materia de aguas, que determinará la concurrencia de títulos competenciales que, a su vez, será fuente de conflictos entre una y otra Administración.

Por todo ello no se puede hablar en materia de aguas en términos exclusivistas, sino que obliga y aconseja establecer los correspondientes meca- 
nismos de colaboración, de forma equilibrada de modo que se pondere debidamente el alcance de los distintos títulos competenciales.

\subsection{Legislación de aguas y la confirmación de la distribución de competencias en ella contenida por el TC}

La Ley de Aguas de 1985 fue dictada observando esta distribución de competencias, al menos desde una perspectiva teórica, y mantenida en la actual legislación del año 2001. El principio general sobre el que actúa es atribuyendo al Estado el gobierno de las cuencas hidrográficas que excedan del ámbito territorial de una sola Comunidad Autónoma (art. 15) y a las CC.AA. el de las cuencas comprendidas íntegramente dentro de su territorio (art. 16). Este criterio actúa igualmente en relación con las aguas subterráneas renovables, atendiendo a su vinculación con las respectivas cuencas. Sin embargo, esta norma fue muy discutida política y doctrinalmente por cuanto se entendía que la misma había producido un exceso en favor de las competencias del Estado. Tras el oportuno recurso de inconstitucionalidad, el TC, en la conocida y estudiada Sentencia 227/1988, de 29 de noviembre, básicamente, ha confirmado la distribución de competencias realizada en la LA del 85 que, como acabamos de ver, parte de la diferenciación entre cuencas intercomunitarias y cuencas intracomunitarias.

\subsection{Las cuencas hidrográficas como criterio esencial de distribución de competencias. Concepto y funcionalidad de la «cuenca hidrográfica»}

La noción de cuenca hidrográfica va a ser la que condicione toda la distribución de competencias en materia de recursos hidráulicos en nuestro país. El agua constituye, evidentemente, una categoría real que presenta una unidad física innegable por encima de compartimentaciones legales y administrativas. Las «cuencas hidrográficas» son las unidades que utiliza la ley para reflejar esta realidad y son definidas por la ley como «el territorio en el que las aguas confluyen en el mar a través de una red de cauces secundarios que convergen en un cauce principal único» y destaca que ésta se considera indivisible a efectos de la gestión del recurso (art. 14).

La cuenca hidrográfica es un concepto capital de la Ley que delimita ámbitos territoriales unitarios e indivisibles en relación con tres cuestiones fundamentales: 
a) el reparto competencial entre el Estado y las CCAA según lo previsto en el artículo 149.1.22 CE;

b) el establecimiento de la planta de la Administración hidráulica (art. 19.3);

c) la planificación hidrológica (art. 38.2).

En definitiva, las cuencas hidrográficas se configuran como unidades de gestión que permiten una administración equilibrada de los recursos hidráulicos que la integran, en atención al conjunto de intereses afectados que, cuando la cuenca se extiende al territorio de más de una CA, son manifiestamente supracomunitarios. Desde un punto de vista técnico es claro también que las aguas de una misma cuenca forman un conjunto integrado que debe ser gestionado de forma homogénea (STC 227/1988) ${ }^{2}$.

Para el TC, desde la lógica de la gestión administrativa, no parece lo más razonable compartimentar el régimen jurídico y la administración de las aguas de cada curso fluvial y sus afluentes en atención a los confines geográficos de cada $\mathrm{CA}$, pues es evidente que los usos y aprovechamientos que se realicen en el territorio de una de ellas condicionan las posibilidades de utilización de los caudales de los mismos cauces, principales y accesorios, cuando atraviesan el centro de otras Comunidades o surten a los cursos fluviales intercomunitarios. Este condicionamiento no sólo se produce aguas arriba en perjuicio de los territorios por los que una corriente desemboca en el mar, sino también aguas abajo, en posible perjuicio de los territorios donde nace o por donde transcurre, y a que la concesión de caudales implica en todo caso el respeto a los derechos preexistentes, de manera que los aprovechamientos concedidos en el tramo inferior o final de un curso pueden impedir o menoscabar las facultades de utilización de las aguas en tramos inferiores. La unidad de cuenca así anunciada recibió un refrendo definitivo por la STC 161/1996, de 17 de octubre, que no vacila en avalar su línea argumental en tal sentido con la apoyatura del mandato constitucional.

Sobre el problema de la delimitación exacta del ámbito geográfico de la cuenca hidrográfica el TC ha precisado la misma sobre la base de dos elementos: la fase final de un río y los afluentes del mismo.

\footnotetext{
${ }^{2}$ Así lo afirma, igualmente, la Carta Europea del Agua, aprobada en 1967 en el seno del Consejo de Europa, donde se declara que la «administración de los recursos hidráulicos debiera encuadrarse más bien en el marco de las cuencas naturales que en el de las fronteras administrativas y políticas». Se trata de un mecanismo que pretende dar respuesta al problema de la gestión del agua en un Estado descentralizado, en que la división territorial en CC.AA. no sirve para resolver cuestiones con transcendencia o incidencia supracomunitaria.
} 
Es lógico que el problema surja cuando se trata de fases finales y afluentes que desembocan en un río principal que, a su vez, discurre por varias Comunidades. El TC parte del concepto de cuenca establecido en el artículo 14 LA. Un concepto que se formula, con carácter indivisible, como unidad de gestión del recurso y por el que debe entenderse «el territorio en el que las aguas fluyen al mar a través de una red de cauces secundarios que convergen en un cauce principal único». Por lo tanto, ni los afluentes ni el tramo final de un río pueden ser objeto de consideración separada.

La gestión de las cuencas hidrográficas cuyo territorio es supracomunitario está encomendada a un organismo singular, las Confederaciones Hidrográficas, lugar de encuentro institucional de la Administración del Estado y las CC.AA., junto con los usuarios en la gestión del agua.

Sobre la base de la cuenca hidrográfica, como anunciamos con anterioridad, la LA del 85 así como el posterior y vigente Texto Refundido de 2001, refrendada en sus planteamientos principales por el TC, parte de atribuir competencia al Estado en materia de recursos hidráulicos sobre las cuencas hidrográficas de carácter intercomunitarias. Es decir, cuando nos encontremos con una cuenca hidrográfica que transcurra por más de una Comunidad Autónoma la competencia corresponderá al Estado. En este caso estamos ante un interés supracomunitario cuya salvaguarda corresponde al Estado. Por el contrario, cuando nos encontremos con una cuenca hidrográfica que transcurra por una única Comunidad Autónoma, al tratarse de una cuenca intracomunitaria, la competencia corresponderá a la Comunidad Autónoma, por cuanto en este caso trata de salvaguardar un interés comunitario.

A pesar de ello, parte de la doctrina critica la interpretación realizada por el TC sobre esta materia. La atribución de competencias exclusivas a las CC.AA. sobre las cuencas intracomunitarias no es conforme con la CE ni con los Estatutos de Autonomía. Para empezar, todos los Estatutos de Autonomía, salvo el de Andalucía, asumieron competencias sobre aprovechamientos hidráulicos (utilización que del agua se puede hacer) y no sobre recursos hidráulicos (el agua misma). Se trata de una diferenciación intencionadamente obviada por el TC a modo de compensación por la atribución en exclusiva al Estado de competencias sobre recursos hidráulicos en cuencas intercomunitarias.

Además, en esta línea crítica, hay suficientes títulos competenciales del Estado en la CE que permitirían una interpretación más estricta de las competencias estatales: bases del régimen energético (art. 149.1.25. ${ }^{\mathrm{a}}$ ), en materia de protección del medio ambiente (art. 149.1.23. ${ }^{a}$ ) o, ya con carác- 
ter exclusivo, sobre las obras públicas de interés general (art. 149.1.24. ${ }^{\mathrm{a}}$ ). Igual conclusión cabe deducir del título competencial del Estado en materia de política económica nacional, habida cuenta del papel, por todos reconocido, que en este campo juegan los recursos hidráulicos. El radical fracaso de una política hidráulica en los últimos años se debe en muy gran medida a la dispersión competencial sancionada en una materia que sobre todo requiere unidad de dirección y ordenación. Toda esta polémica responde en nuestro país a una situación en la que existe una clara tendencia a marginar y romper todo lo que pueda responder a una idea de generalidad, de unión; de unidad. Son los particularismos los que priman y dividen; la fragmentación. Y ello no es bueno con carácter general y, por ende, tampoco en materia de los recursos hidráulicos. Las aguas son habitualmente fuente de tensiones. No obstante, constituyen asimismo vínculos de unión importantes. Expresión de planteamientos globalizados y generales, únicos, por otra parte, que permiten el aprovechamiento racional y exhaustivo de los recursos hidráulicos.

Este reclamo de un mayor protagonismo competencial del Estado no debe significar ignorar los intereses de las CC.AA. en la gestión de los recursos hidráulicos. Así, las CC.AA. tienen una muy importante presencia en las Confederaciones Hidrográficas, órganos descentralizados de la Administración estatal, lugar donde pueden y deben defender sus intereses autonómicos. El propio TC, sobre este punto, ha señalado, tras insistir en la idea de que la integración en los órganos de gobierno de las correspondientes Confederaciones Hidrográficas es el «modo más directo» de incidir en los intereses afectados por la gestión del agua, señala que las actuaciones que las CC.AA. pueden realizar directamente en las cuencas supracomunitarias son complemento de aquella participación orgánica y sólo son factibles en la medida en que no interfiera la actuación de la Confederación Hidrográfica ni la perturbe.

El reto, además, está en poner en funcionamiento el juego de los mecanismos de cooperación y colaboración.

\subsection{La legislación sectorial de las CC.AA. y su interrelación con la competencia estatal en materia de aguas}

A pesar de que, como hemos visto, el principio general es el de la competencia estatal sobre las cuencas hidrográficas intercomunitarias, las CC.AA. tienen la posibilidad de incidir en su regulación de una forma indirecta, es decir, a través de títulos competenciales sectoriales con incidencia en la materia de aguas. 
El examen de la normativa sectorial de las CC.AA. con incidencia en esta materia pone de manifiesto que muy diversas actividades o usos que tienen algún punto de conexión con el agua o con el dominio público hidráulico, quedan sometidas a un preceptivo trámite de informe (frecuentemente favorable y vinculante) o autorización de la respectiva Comunidad Autónoma (por ejemplo, en materia de zonas húmedas, espacios naturales protegidos, fauna fluvial, actividades clasificadas, urbanismo, restauración hidrológico-forestal). Esta intervención administrativa autonómica (informes o autorizaciones) se superpone y añade a la preceptiva autorización o concesión que en las cuencas supracomunitarias corresponde a las Confederaciones Hidrográficas. Ello significa que de no obtenerse las dos autorizaciones requeridas la actividad o uso no podrían realizarse. Desde la perspectiva de la Administración hidráulica competente para la gestión del agua en las cuencas supracomunitarias, nos encontramos con la paradoja de que el título competencial específico y directo (global y genérico) sobre los recursos hídricos puede quedar desplazado por el título competencial sectorial (indirecto y parcial) ejercido por una Comunidad Autónoma. Por otro lado, el ejercicio de una competencia sectorial autonómica que tiene una limitación territorial inherente (el ámbito de la respectiva Comunidad Autónoma), produce consecuencias supraterritoriales que interfieren en la competencia sobre la gestión del agua en las cuencas supracomunitarias.

No parece que sea deseable que tratándose del ejercicio de competencias administrativas distintas pero que inciden en un mismo espacio físico, discurran de manera paralela e independiente sin posibilidad de integración. Es necesario buscar formas de integración que nos ofrece el ordenamiento jurídico.

Por tanto, insistimos, independientemente del criterio esencial de la cuenca hidrográfica que constituye el criterio fundamental para la distribución de competencias entre Estado y CC.AA. en materia de aguas, existen, además, múltiples títulos competenciales con incidencia sectorial en la gestión del agua, lo que motiva que el protagonismo regulador se diversifique afectando a todas las Administraciones de base territorial. En este sentido, y ante la irremediable amplitud reguladora que se produce, con el fin de evitar los tradicionales problemas debidos a la superposición práctica de los distintos títulos competenciales, es necesaria la coordinación y colaboración entre las Administraciones actuantes. El T.C. llega a la conclusión de que la «atribución de una competencia sobre un ámbito físico determinado no impide necesariamente que se ejerzan otras competencias en ese espacio, siempre que ambas tengan distinto objeto jurídico, y que el 
ejercicio de las competencias autonómicas no interfieran o perturben el de las estatales, por lo que, frecuentemente, resultará imprescindible el establecimiento de mecanismos de colaboración que permitan la necesaria coordinación y cooperación entre las Administraciones Públicas implicadas» ${ }^{3}$.

Ante semejante telaraña de competencias que se interrelacionan y condicionan unas a otras, es necesario recordar la doctrina del TC que insiste en la colaboración como técnica para superar los conflictos que puedan surgir entre la Administración estatal y autonómica a la hora de determinar cómo debe ser la gestión del agua en España, pues sólo arbitrando sistemas de colaboración orgánica o procedimental se puede lograr la coherencia en la actuación administrativa. Estas mismas pautas deben ser aplicadas a las relaciones entre las Administraciones autonómicas y las Administraciones locales, pues también entre ellas deben evitarse los conflictos competenciales.

\section{Competencias de la Administración local en materia de recursos hidráulicos}

\subsection{Introducción}

Antes de sistematizar las competencias que en materia de aguas o recursos hidráulicos tiene la Administración local, es necesario tener en cuenta el limitado papel que a dicha Administración le corresponde desempeñar. La razón es obvia, como hemos ido viendo, el problema de la gestión de los recursos hidráulicos tiene un interés que, normalmente, va a superar el ámbito local, con frecuencia será comunitario o, en muchas ocasiones, su trascendencia será intercomunitaria. Razón por la que será en las instancias territoriales superiores donde se decidan las cuestiones más importantes que afectan a esta materia.

Además, no teniendo la Administración local capacidad legislativa en sentido estricto, el ámbito normativo sobre recursos hidráulicos queda, obviamente, al margen de las competencias de esta Administración territorial.

Un reflejo de esta idea lo acusamos en las pocas referencias que a la Administración local hace la LA. Tan sólo se menciona, como veremos, en relación al concesionario en los abastecimientos a varias poblaciones. No

\footnotetext{
${ }^{3}$ SSTC 15/1998, de 22 de Enero y 110/1998.
} 
obstante, la legislación básica reguladora del régimen local en España ${ }^{4}$ reconoce ámbitos competenciales en materia de aguas.

El artículo 25 LRBRL que enumera una serie de materias sobre las que necesariamente la Ley —estatal o autonómica - tendrá que reconocer competencias a la Administración local, en su letra l) alude al «suministro de agua..., alcantarillado y tratamiento de aguas residuales».

El artículo 26 LRBRL, encargado de describir una serie de servicios mínimos obligatorios, señala como competencia obligatoria en todos los municipios el abastecimiento domiciliario de agua potable y el alcantarillado.

Por otro lado, en el artículo 86.3 LRBRL encargado de declarar la reserva en favor de las Entidades Locales de una serie de actividades o servicios esenciales, es decir, tiene lugar la publicatio o declaración de una serie de servicios públicos, se alude al abastecimiento y depuración de aguas.

Se trata, en definitiva, del suministro o abastecimiento domiciliario de agua potable y la depuración de aguas residuales de dos servicios que tradicionalmente aparecen en la legislación íntimamente ligados a la Administración local. Sin embargo, como luego se dirá, este servicio tradicionalmente local, está viviendo una especie de desapoderamiento por parte, fundamentalmente, de las Comunidades Autónomas que utilizan sus diversos títulos competenciales sectoriales para recortar las competencias locales sobre la materia.

Todavía en el ámbito local, pero refiriéndonos a la Administración provincial, destacar que el artículo 36.1 b) LRBRL atribuye a las Diputaciones provinciales garantizar los principios de solidaridad y equilibrio intermunicipales, en el marco de la política económica y social y, en particular:

a) Asegurar la prestación integral y adecuada en la totalidad del territorio provincial de los servicios de competencia municipal.

b) Participar en la coordinación de la Administración local con la de la Comunidad Autónoma y la del Estado y son competencias propias de la Diputación las que les atribuyan, en este concepto, las leyes del Estado y de las Comunidades Autónomas en los diferentes sectores de la acción pública y, en todo caso:

\footnotetext{
${ }^{4}$ Ley Reguladora de las Bases del Régimen Local (LRBRL), 7/1985, de 2 de abril; Texto Refundido de las Disposiciones legales vigentes en materia de régimen local, R.D. Leg. 781/1986, de 18 de abril (TRRL).
} 
- La coordinación de los servicios municipales entre sí para la garantía de la prestación integral y adecuada a que se refiere el ap. a) núm. 2 del artículo 31.

- La prestación de servicios públicos de carácter supramunicipal y, en su caso, supracomarcal».

\subsection{El servicio de abastecimiento de agua a poblaciones}

Se trata de dos servicios de capital importancia para el normal desarrollo de la vida. Desde otra perspectiva, las aguas para abastecimiento de población tienen unas exigencias de calidad y de garantía sanitaria que no deben poseer en la misma medida las aguas que van a ser destinadas a otros usos.

La preeminencia que, en general, tiene el abastecimiento de aguas a poblaciones en nuestro ordenamiento jurídico, se reconoce expresamente en la LA. Así, en primer lugar, dentro de la ordenación de usos a que se refiere el artículo 60 de la LA aparece en primer lugar el abastecimiento de población. Por cierto, en este precepto se da una definición de lo que se entiende por abastecimiento de poblaciones, y que no se limita a lo que podríamos comprender como «agua de boca» sino que se extiende a pequeños usos industriales caracterizados, sobre todo, por estar conectados con la red municipal ${ }^{5}$.

La atribución de aguas para abastecimiento de poblaciones, como para el resto de los usos, se realizará normalmente mediante concesión, sujeta a plazo y que se extingue por su transcurso.

Así, aunque la titularidad del servicio corresponda a los Ayuntamientos, se trata de un servicio público cuya existencia o efectiva prestación depende de voluntades ajenas a la propia Corporación titular del servicio, la de la autoridad titular del bien sobre el que actúa el servicio.

\subsection{El abastecimiento a poblaciones como servicio público}

Analicemos más detenidamente la consideración como servicio público del abastecimiento de poblaciones. Este bloque está constituido, como

\footnotetext{
${ }^{5}$ Expresamente dice el apartado tercero de este artículo que el abastecimiento de población incluye «en su dotación la necesaria para industrias de poco consumo de agua situadas en los núcleos de población y conectadas a la red municipal».
} 
hemos visto, fundamentalmente por la legislación de régimen local (LRBRL como cabecera del ordenamiento) con la adición de algunas normas autonómicas.

Los principales problemas jurídicos que se plantean son los referidos a la misma determinación de las competencias locales y autonómicas, a la elección de las formas organizativas de prestación del servicio y, finalmente, a la tarifa como tema anexo al de la repercusión de los costes al usuario del servicio.

El artículo 25.2 1) LRBRL dispone que el municipio ejercerá competencias en los términos de la legislación del Estado y de las CC.AA. en materia de «suministro de agua...». Este suministro de agua es, además, un servicio que debe prestarse indefectiblemente en cada municipio [art. 26.1 a) LRBRL], siendo declarado, por último, como servicio de carácter esencial reservado a las entidades locales en el artículo 86.3 LRBRL.

El Estado puede regular esta materia del abastecimiento a poblaciones utilizando títulos constitucionales como la titularidad del dominio público y de la ordenación de usos y de obras hidráulicas de interés general vinculadas a la realización efectiva de esos usos (art. 149.1.22. ${ }^{a} \mathrm{CE}$ ); el aspecto sanitario de las aguas, materia en que al Estado le corresponde la fijación de la normativa básica (art. 149.1.16. ${ }^{\mathrm{a}} \mathrm{CE}$ ), o la competencia para fijar las bases en materia de medio ambiente (art. 149.1.23. ${ }^{\mathrm{a}} \mathrm{CE}$ ).

Las CC.AA., por su parte, pueden intervenir a partir de la capacidad de legislar en el ámbito de las obras públicas de su interés (art. 148.1.4. ${ }^{a} \mathrm{CE}$ ) o de los proyectos, construcción y aprovechamientos hidráulicos de su interés (art. 148.1.10 a ${ }^{a} \mathrm{CE}$ ) y, por supuesto, en desarrollo de la legislación básica estatal, sea ésta de aguas, de régimen local, de sanidad o medioambiental.

Así, a pesar de que la dicción de la LRBRL pudiera deducirse que este servicio de abastecimiento domiciliario de agua quedaba reservado a la competencia de las entidades locales, lo cierto es que tanto el Estado como las CC.AA. pueden legislar sobre la materia, todo ello debido a la evidente «supramunicipalidad» de muchas actuaciones necesarias para el abastecimiento a poblaciones.

Con carácter meramente ejemplificativo, la Comunidad de Madrid asume el servicio de aducción que comprende las labores de captación y alumbramiento, embalse, conducciones por arterias o tuberías primarias, tratamiento y depósito (art. 1.2). Por su parte, a los municipios les corres- 
ponde el servicio de distribución (art. 2). De otro lado, esta Ley madrileña también considera como competencia de interés de la Comunidad los servicios de depuración, dejando a los municipios las funciones de alcantarillado ${ }^{6}$. Muy parecido al modelo madrileño es el de la Ley asturiana de $1994^{7}$. Algo más compleja es la legislación catalana. La vigente Ley 6/1999, de 12 de julio, sobre ordenación, gestión y tributación del agua, dispone que corresponde a la Generalidad de Cataluña la determinación de la política de abastecimiento y de saneamiento de las aguas y la coordinación de las Administraciones competentes, así como la promoción y la ejecución, en caso de ser necesario, de las actuaciones de política hidrológica necesarias para paliar los déficit y los desequilibrios en Cataluña. Por otro lado, atribuye a las entidades locales determinadas competencias, de acuerdo con la normativa de régimen local, con la normativa de sanidad y con lo que prevé la propia ley. La administración local del agua se configura en esta Ley con la creación de las llamadas Entidades locales del agua (ELA), definidas como entes locales o agrupaciones de entes locales con personalidad jurídica propia y capacidad para gestionar uno o más sistemas públicos de saneamiento de aguas residuales y el sistema o sistemas de abastecimiento de agua en alta y de suministro de agua en baja de los municipios que lo componen ${ }^{8}$.

${ }^{6}$ Véase la Ley de la Comunidad de Madrid 17/1984, de 20 de diciembre (ref. Ar. LCM 1984/3.118). En ella se refleja claramente una nueva consideración del abastecimiento y saneamiento, y, tal y como expresa su exposición de motivos, establece una regulación de los mismos «en función de los ámbitos territoriales que resulten afectados». Por otra parte, considera de interés supramunicipal «aquellos servicios cuya prestación exige la superación de los límites del término municipal o que tienen evidentes repercusiones fuera de ellos. La aducción o traída de aguas incluso embalses captaciones y grandes redes puede precisar el recurso a otros ámbitos para encontrar las condiciones exigidas para un buen abastecimiento; igualmente la depuración de aguas residuales incide en la contaminación del agua que circula por los términos situados aguas abajo, cuando funciona mal o deja de funcionar. Por ello los servicios de aducción y depuración son considerados de interés para la Comunidad de Madrid». Mientras que, por el contrario, se reconoce y potencia el interés municipal «en los servicios relativos a la distribución de agua desde los depósitos a las acometidas y en los servicios de alcantarillado o recogida de aguas residuales hasta la depuradora». Asimismo, insiste en la «conveniencia de una gestión unitaria de los servicios de interés de la Comunidad y la existencia en Madrid de un organismo como el Canal de Isabel II, de gran implantación en la región y con más de un siglo de experiencia en abastecimiento de agua, y con funciones reconocidas, en depuración de aguas residuales, aconsejan la implantación de la gestión integrada de los servicios de aducción y depuración, extendiendo sus funciones y su ámbito territorial para una eficaz explotación de ambos servicios».

${ }^{7}$ Ley 1/1994, de 21 de febrero, de abastecimiento y saneamiento de aguas (ref. Ar. LPAS 1994/38).

${ }^{8}$ Las Entidades Locales del Agua (ELA) son órganos de cooperación entre las entidades locales y la Agencia Catalana del Agua, cuya creación está prevista en la Ley 6/1999, para una mejor gestión de los recursos hídricos y de las obras hidráulicas y para la prestación de servicios relacionados con el agua. Puede tratarse de un ente local (un municipio, una comarca, una entidad municipal descentralizada, una entidad metropolitana, una mancomunidad de municipios...) o una 
En cualquier caso, el servicio de abastecimiento podrá ser prestado por los municipios conforme a cualquiera de las fórmulas de gestión previstas en la LRBRL. No presenta este servicio ninguna excepción en cuanto a la posibilidad de gestión directa o indirecta.

Lo que conviene advertir es que cada vez es mayor la presencia de organizaciones supramunicipales para propiciar la gestión del servicio como prueba de la complejidad progresivamente mayor del abastecimiento a poblaciones y de la continua aparición de elementos de supramunicipalidad en la misma. En tal sentido es muy usual la utilización por los municipios de la figura de las Mancomunidades municipales (sí es una entidad local; unión de diferentes municipios; art. 44 LRBRL, 35 y 36 TRRL) y también la del consorcio (no es entidad local sino forma de prestación de los servicios públicos; unión de diferentes Administraciones e incluso con personas jurídico privadas sin ánimo de lucro; arts. 87 LRBRL, 110 TRRL y 7 LPC).

\section{FORMAS DE GESTIÓN DEL SERVICIO PÚBLICO DE ABASTECIMIENTO DE AGUA POTABLE A VARIOS MUNICIPIOS}

\section{Introducción}

Las Entidades locales, de base territorial, tienen reconocida constitucionalmente, autonomía para la gestión de sus intereses. Esta autonomía, entendida como derecho y capacidad efectiva para ordenar y gestionar una parte importante de los asuntos públicos, en el marco de la Ley (estatal y autonómica), bajo su propia responsabilidad y en beneficio de sus habitantes constituye una garantía institucional según lo establecido en la doctrina del Tribunal Constitucional a partir de la STC 32/19819. Ello supone, tal y como establece, entre otras, la STS de 18 de julio de 1997, «por una parte, la atribución de una esfera de intereses propios y de competencias para su gestión y, por otra, el reconocimiento de una capacidad de autogestión y de una capacidad para representar y gobernar los intereses de las comunidades que constituyen las entidades locales, impidiendo la existencia de determinados controles de otras Administraciones Públicas».

\footnotetext{
agrupación de entidades locales con personalidad jurídica propia (un consorcio), capacitada para gestionar íntegramente uno o más sistemas públicos de saneamiento de aguas residuales y/o el sistema o sistemas de suministro de agua de los municipios que la componen. De modo que puede haber ELA básicas para el saneamiento, ELA básicas para el suministro y ELA básicas mixtas que gestionen tanto el saneamiento como el suministro de agua de su ámbito.
}

${ }^{9}$ Reiterada después, entre otras, en SSTC 38/1983, 27/1987, 76/1988, 213/1988. 
Pues bien, de acuerdo con ello, existe un principio de libre elección del modo de gestión de los servicios públicos en el ámbito local, que se deduce del artículo 85.2. LRBRL, a salvo la reserva de la gestión directa de los servicios que impliquen el ejercicio de autoridad.

Se permite entonces las diversas fórmulas asociativas que ofrece la legislación de régimen local, distinguiendo entre las formas de gestión directas y las formas de gestión indirectas. Y junto a ellas, la gestión conjunta de medios instrumentales de los servicios de las entidades locales, a través de la colaboración intermunicipal y la cooperación de las distintas Administraciones Públicas, siempre y cuando se respeten los postulados constitucionales y los condicionamientos impuestos por el Ordenamiento Jurídico, especialmente en lo relativo al diseño de las propias competencias y el respeto a la autonomía local ${ }^{10}$.

\section{Alternativas de gestión para la prestación del servicio público de abastecimiento a varias poblaciones: artículo 89 LA}

En el primer apartado hemos visto que, de acuerdo con el artículo 89 de la Ley de Aguas, el abastecimiento de agua potable a varios municipios puede llevarse a cabo de dos formas. La primera de ellas a través de la constitución de los municipios afectados en una Mancomunidad o consorcio o entidad semejante. Y que no plantea más problemas que el de la necesaria cesión de competencias de cada uno de los municipios al ente supramunicipal para la gestión de un servicio común, y le necesidad de llegar a un acuerdo con la rapidez deseada en estos casos. La segunda alternativa, que elude el requisito de la obligatoria constitución en un ente supramunicipal, es que el servicio sea prestado por una misma empresa concesionaria. Teniendo en cuenta cuáles son las formas de gestión de los servicios públicos locales con carácter general, precisemos, a continuación, de qué manera puede realizarse la prestación del servicio en cuestión por una misma empresa concesionaria y qué tipo de gestión es, lo que nos va a llevar a analizar uno de los problemas actuales relacionado con la iniciativa pública local económica a través de empresas de capital público o mixto.

\subsection{Constitución de los Municipios afectados en Mancomunidad, Consorcio o Entidades semejantes}

Ésta sería la forma jurídica más sencilla para proceder a la prestación del servicio de suministro de agua potable a varios municipios. Esto es, tal

${ }^{10}$ Véase, entre otras, la STS de 18 de julio de 1997, Ar. 6.123, F.J. 2. 
y como prevé el artículo citado de la Ley de Aguas, la constitución de las Corporaciones locales afectadas en Mancomunidades o consorcios u otras entidades semejantes para la solicitud y obtención de otorgamiento de concesiones para abastecimiento de agua a varias poblaciones.

La mancomunidad de municipios es una asociación de los municipios afectados, precisamente por la necesidad de gestionar el servicio en un ámbito más amplio que el de un municipio aislado. Las funciones que les corresponderían serían las derivadas de asumir la gestión de la parte del servicio que les sea transferida. Obviamente serán aquellos elementos, comunes a los distintos municipios mancomunados (recursos, red de distribución mancomunal, potabilización conjunta, etc.), los que deberá de gestionar propiamente la mancomunidad, aunque, si los municipios lo acordaren, no existiría dificultad alguna para que incluso asumiera, en su ámbito, la distribución domiciliaria ${ }^{11}$.

La norma fundamental de estos entes es su Estatuto, en el que debe constar la denominación de la Mancomunidad, su ámbito territorial, su objeto o finalidad, las competencias que se le atribuyen, órganos de gobierno (representativos de los Ayuntamientos mancomunados), reglas de funcionamiento, régimen de recursos económicos y plazo de duración de la Entidad. La Mancomunidad tiene personalidad jurídica propia e independiente de la de los municipios que la crean. El proceso de su creación se regula por la legislación autonómica, aunque el artículo 44 LRBRL fija algunos criterios básicos relativos a la aprobación de los Estatutos. El proyecto de Estatutos se somete a informe de la Diputación o Diputaciones correspondientes y, finalmente, los Plenos de todos los Ayuntamientos deben aprobar el Estatuto ${ }^{12}$.

Por otra parte, la figura del consorcio es administrativamente la que corresponde en aquellos casos en que, para la gestión del servicio, se asocian organismos de la Administración de distinto nivel. Sus funciones pueden ser todas aquellas que le deleguen los organismos consorciados, pudiendo llegar a gestionar el servicio en toda su integridad. Los consorcios son entidades, dotadas de personalidad jurídica, que constituyen diversos Entes Públicos de naturaleza territorial o institucional que pertenecen a escalones de administración territorial diferentes (estatal, autonómico o local); aunque también se prevé en la legislación local que se puedan inte-

\footnotetext{
${ }^{11}$ Véase C. GonZÁlez-Antón Álvarez, El uso del agua por las Administraciones Locales, op. cit., pp. 32 y ss.

12 Véase L. Cosculluela Montaner, Manual de Derecho Administrativo I, Civitas, Madrid, 2002, p. 271.
} 
grar en ellos entidades privadas. Surgen para la realización de fines de interés común, que normalmente se concretan en la gestión de servicios, de bienes, o de obras, que entran en la competencia de los diversos Entes Públi$\cos$ que integran el Consorcio. Por ello tienen una clara finalidad de coordinación y cooperación que se resuelve en una nueva estructura organizativa a la que, en cualquier caso, se le va a encomendar la realización por ella misma de aquellas actividades relacionadas con competencias compartidas o concurrentes de los Entes Públicos que constituyen el Consorcio, buscando la optimización de las prestaciones a realizar.

El artículo 57 LRBRL se refiere a los consorcios y convenios administrativos como instrumentos de cooperación económica, técnica y administrativa entre la Administración Local y las Administraciones del Estado y de las CC.AA. Estos convenios tienen el mismo significado que en el ámbito de las relaciones interadministrativas entre entidades locales y a ellos les es de aplicación el principio de que en ningún caso pueden suponer alteración o renuncia de las propias competencias legalmente atribuidas, como tampoco puede implicar la atribución de facultades administrativas que, como la de coordinación de las entidades locales, ha de estar necesariamente conferida por la Ley, ya que no es una materia disponible para las Administraciones Públicas ${ }^{13}$.

Cualquiera de estas dos opciones sería jurídicamente correcta para los objetivos de prestación del servicio público de suministro de agua a diversos municipios. Además, en algunos casos, podría constituir un primer paso para la definitiva creación de un Área Metropolitana, como Entidad supramunicipal para la gestión de competencias y servicios comunes a los municipios integrados en las grandes conurbaciones urbanas. La LRBRL prevé en el artículo 43 la creación por las CC.AA. de Áreas Metropolitanas, que define como «entidades locales integradas por los municipios de grandes aglomeraciones urbanas entre cuyos núcleos de población existan vinculaciones económicas y sociales que hagan necesaria la planificación conjunta y la coordinación de determinados servicios». Su regulación se remite a la legislación autonómica, imponiendo tan sólo que todos los municipios del Área estén representados en sus órganos de gobierno.

Cualquiera de las opciones de constitución de entidades supramunicipales para la prestación de este servicio esencial evitaría muchos de los

\footnotetext{
13 Este límite lo recoge el artículo 8 de la L. 30/1992, cuyas previsiones para los convenios de colaboración —Estado y CC.AA. — contenidas también en el art. 6 sirven para integrar como derecho supletorio el régimen de los convenios de colaboración de las entidades locales.
}

Expresiva es, en este aspecto, la Sentencia del Tribunal Supremo de 18 de julio de 1997, Ar. 6.123. 
conflictos jurídicos que de otra forma, sin duda alguna, tienden a aparecer. Sin embargo, aun siendo ésta la forma jurídica más adecuada y acorde con el principio de coordinación y gestión integral del agua cumpliendo con las aspiraciones de la ley que lo desarrolla como cauce natural, presenta la desventaja del largo periodo de tiempo que puede transcurrir durante la tramitación del correspondiente procedimiento de constitución, teniendo en cuenta el número de municipios que se encuentren afectados y cuyas formas políticas de organización y gestión pueden ser, y de hecho serán, bien distintas. Es por ello por lo que la búsqueda de otras soluciones se hace imprescindible, mientras la deseada constitución de una entidad supramunicipal no tenga lugar.

\subsection{El servicio de abastecimiento de agua potable a varios municipios prestado por una misma empresa concesionaria}

La segunda de las opciones que permite el artículo 89.1 para el abastecimiento a varias poblaciones es la de que el servicio sea prestado por una misma empresa concesionaria. En este caso, lógicamente, todos los municipios afectados deberían formalizar la adjudicación de su servicio de suministro de agua potable a la misma empresa. Dentro de esta posibilidad se plantean las siguientes alternativas:

\subsubsection{Creación por todos los municipios afectados de una nueva empresa de aguas}

Los municipios afectados podrían proceder a la creación de una nueva empresa de aguas para la prestación del servicio de abastecimiento de agua potable del que son titulares en sus respectivos términos municipales estando dicha empresa participada por cada uno de los municipios afectados. Se trataría de una empresa pública enteramente o de capital mixto, lo más probable.

2.2.2. Prestación del servicio por la empresa de aguas que presta dicho servicio en el municipio económicamente más importante ${ }^{14}$ : la conversión de un servicio público local de carácter esencial en una actividad competitiva

Nos encontramos en el supuesto de que el municipio principal y más importante desde el punto de vista territorial, poblacional y económico,

\footnotetext{
${ }^{14}$ Municipio al que llamaremos municipio principal.
} 
presta el servicio público de abastecimiento de agua potable a través de una empresa, sociedad anónima, de capital público. Dicho municipio dispone de una concesión de aguas públicas otorgada por la Confederación Hidrográfica correspondiente, para destino a uso de abastecimiento de agua potable exclusivamente para la población de su término municipal. Otros municipios vecinos pretenden que el servicio de abastecimiento de agua potable en sus respectivos términos municipales sea prestado por esta misma empresa acogiéndose a la segunda de las posibilidades que plantea el artículo 89 de la LA, sin que para ello tengan que constituir una entidad supramunicipal, como hemos señalado con anterioridad. La razón fundamental radica exclusivamente en las mejores tecnologías disponibles y aplicadas por esta empresa para la prestación del servicio, lo que supone un ahorro de costes para estos municipios al tiempo que permite la prestación de un servicio esencial en las condiciones de cantidad y calidad exigidas legalmente.

Jurídicamente, caben distintas opciones. Una de ellas es que el Ayuntamiento principal como titular de una concesión de aprovechamiento de aguas procedentes de una fuente determinada, para abastecimiento de la ciudad, solicite la modificación de las características de la concesión con el fin de destinar parte del caudal al abastecimiento de agua potable a otros municipios, a través de su empresa de aguas.

Los argumentos que puedan sustentar esta iniciativa pueden basarse, según los casos, en la situación de precariedad que, temporal o permanentemente, se da en los sistemas de abastecimiento de esos otros municipios vecinos, en los que los ciudadanos usan aguas de mala calidad e incluso de calidad no potable en determinados casos y períodos de tiempo. Siempre y cuando se acredite que el sistema por el que se abastece el municipio principal permite asegurar las necesidades tanto de los abastecimientos como de los regadíos con un uso racional y eficaz del agua, podría entenderse que se logra de este modo satisfacer un interés público atendiendo al principio de unidad de gestión, economía del agua y tratamiento integral de la misma.

Esta posibilidad exige un compromiso formalizado entre el Ayuntamiento principal y los municipios afectados, compromiso que acreditaría que estos municipios están dispuestos a que se suministre agua potable con las aguas inicialmente adscritas al municipio principal exclusivamente. Con este compromiso formalizado no se produciría invasión de las competencias de cada uno de los municipios afectados. La titularidad del servicio de suministro de agua potable seguiría siendo, en todo caso, titularidad de cada municipio que, simplemente, solicitan ser abastecidos con 
aguas de calidad y que este abastecimiento tenga lugar por una misma empresa.

Una vez modificada la concesión de aguas del municipio principal, estaría abierta la posibilidad de que la empresa que actualmente presta el servicio de abastecimiento domiciliario de agua potable en dicho municipio realice el mismo servicio en el resto de municipios afectados.

Si bien, se plantean algunos problemas que es necesario destacar:

a) El objeto social de la empresa de aguas del municipio principal.

La primera de las cuestiones a tener en cuenta en este supuesto es la que tiene que ver con los estatutos de dicha empresa de aguas que, normalmente, adoptará la forma de sociedad anónima. Sería necesario comprobar hasta qué punto está previsto estatutariamente que en el objeto social de esta empresa se pueda prestar a municipios diferentes del principal.

b) El carácter público y esencial del servicio y su prestación más allá de los límites del término municipal: la conversión del servicio público en actividad competitiva.

Salvado este primer escollo, nos encontramos con otro problema que deriva del propio carácter del servicio que se pretende prestar más allá del ámbito territorial en el que el Ayuntamiento principal ejerce sus competencias, entre las que se encuentra el abastecimiento de agua potable a la ciudad.

El servicio de abastecimiento de agua potable en el municipio principal está adjudicado a una empresa, total o parcialmente pública. Con la modificación de la concesión se pretende que esa misma empresa continúe prestando el servicio a los municipios. Si bien, esta extensión del servicio no puede tener lugar automáticamente sino que requiere el oportuno procedimiento administrativo. Además, el carácter de servicio público desaparece en el sentido que a continuación se expone, y pasa a convertirse en una actividad competitiva y sometida a los principios de la contratación pública.

b.1) Diferenciación entre prestación directa y prestación indirecta del servicio público de abastecimiento de agua potable.

En primer lugar, debemos establecer la diferencia entre, de un lado, la prestación directa de un servicio público por medio de una sociedad de Derecho privado en cuyo capital es mayoritaria la participación de la 
Administración o empresa mixta (art. 155.2 LCAP) dentro del mismo ámbito geográfico al que alcanzan las potestades de esa Administración Local (se trata del supuesto de abastecimiento de agua potable en el municipio principal por su empresa de aguas); y, de otro lado, el servicio de prestación directa del mismo servicio público fuera de ese espacio territorial (es el supuesto tras la modificación de la concesión del Ayuntamiento principal con el fin de que la misma empresa de aguas preste el servicio a los otros municipios vecinos).

Esta diferenciación es del todo necesaria, pues de lo dicho se desprende que no se trata de actos administrativos idénticos, en el sentido de que la prestación del servicio de abastecimiento de agua potable a esos otros municipios vecinos no va a ser prestado por una persona jurídica en la que tales Administraciones Locales dispongan de la mayoría en el número de sus participaciones sociales, sino que va a ser prestado por una tercera persona jurídica (empresa de aguas del Ayuntamiento principal) que cuenta con ese nivel participativo pero a favor del Ayuntamiento principal.

Esto quiere decir que el abastecimiento de agua potable a los municipios vecinos escapa del concepto de gestión directa del servicio público por parte de la Administración Local titular del mismo y entra en el concepto de gestión indirecta de éste por un tercero. Y esto significa en términos prácticos y legales que la empresa de aguas del Ayuntamiento principal pierde los rasgos singulares que le son característicos para entrar en el mercado como una entidad más que trata de diseñar su volumen negocial por el cauce de un incremento en la prestación de servicios públicos objetivo tendencial explícito de la sociedad que le pueden ser adjudicados.

b.2.) El convenio interadministrativo para la prestación del servicio público de abastecimiento de agua potable a varios municipios a través de la empresa de aguas del Ayuntamiento principal no es la forma jurídica correcta.

Esta alternativa que venimos comentando normalmente se ha llevado a la práctica a través de la figura del convenio interadministrativo entre el municipio principal y los municipios vecinos afectados, con el fin de determinar que la prestación del servicio de abastecimiento domiciliario se lleve a cabo por la empresa de aguas del Ayuntamiento principal. Esta forma de proceder, como es lógico, ha producido los consecuentes conflictos jurídicos e impugnaciones de los citados convenios ante la Jurisdicción contencioso-administrativa, toda vez que con ello se infringen los principios de publicidad y concurrencia en la adjudicación de los servicios 
públicos conforme a lo establecido en la Ley de Contratos de las Administraciones Públicas.

Y es que en este supuesto no se trata ya de la prestación de un servicio público esencial del propio Ayuntamiento principal dentro de su término municipal, sino de la extensión de este servicio a otros municipios, con lo que realmente lo que se produce es una ampliación del ejercicio de una pura actividad económica prestada por una empresa que está sometida a las normas del Derecho privado. De manera que a través de la figura del convenio administrativo se están vulnerando las exigencias de libre concurrencia y publicidad que fija la normativa de contratación de Derecho público. Y, aun cuando los convenios interadministrativos están excluidos del ámbito de aplicación de la Ley de Contratos, no obstante, el supuesto en cuestión supone una exclusión ilícita de la normativa legal aplicable en materia de contratación pública: libre concurrencia a la prestación del servicio si no se opta por la prestación directa del mismo a través del propio Ente público sea por sí mismo o por medio de la constitución de una persona pública o privada interpuesta.

La jurisprudencia ha tenido oportunidad de pronunciarse sobre este tema. Así, la Sentencia del Tribunal Superior de Justicia de Valencia de 3 de julio de $2002^{15}$, en la que se declara la nulidad de un convenio interadministrativo entre dos Ayuntamientos y una sociedad de economía mixta para la gestión indirecta y prestación del servicio público de agua potable y alcantarillado de uno de los municipios a través de dicha socie$\mathrm{dad}^{16}$. El argumento principal consiste en la vulneración de los principios de publicidad y libre concurrencia que imponen las normas de contratación pública y que se eluden, en este caso de manera ilícita, a través de la figura del convenio interadministrativo, a pesar de que éste se excluye del

\footnotetext{
15 Ref. La Ley 2003, 10.866.

${ }^{16}$ Se trataba del Convenio interadministrativo suscrito entre los Ayuntamientos de Xirivella y de Torrent para la prestación del servicio público de abastecimiento de agua y de alcantarillado a través de la empresa Aigües de l'Horta, S.A., empresa participada mayoritariamente por el Ayuntamiento de Torrent. Con lo que no se trata de una forma de gestión directa del servicio, sino de una gestión indirecta del servicio a través de «sociedad mercantil y cooperativas legalmente constituidas cuyo capital social sólo parcialmente pertenezca a la Entidad Local» (art. 85 LRBRL). En el caso concreto, la empresa Aigües de l'Horta no tiene ninguna relación con el Ayuntamiento de Xirivella. La participación mayoritaria del Ayuntamiento de Torrent tiene relevancia sólo cuando se trata de prestar el servicio dentro del término municipal de Torrent, pero no cuando se pretende extender más allá de sus límites territoriales. En este caso, la empresa entra en el mercado como una entidad más en régimen de competencia, que pretende ampliar sus beneficios a través de la prestación de servicios públicos en otros municipios. Impugna este convenio Aguas de Valencia, S.A., como clara interesada en la prestación de ese mismo servicio mediante la adjudicación del mismo siguiendo el procedimiento y principios de la contratación pública.
} 
ámbito de aplicación de la Ley de Contratos de las Administraciones Públicas. Los Ayuntamientos afectados apoyan su actuación en la potestad de autoorganización que les corresponde, de manera que, una vez establecida una actividad económica que reviste el carácter de servicio público, el Ayuntamiento puede extenderla a otros municipios si lo considera oportuno, con base en la cooperación administrativa que forma parte de su potestad de autoorganización y sin que ello suponga la ampliación del ejercicio de una actividad económica, sino que más bien se trata de la prestación de su servicio a otro municipio en régimen de cooperación. El Tribunal rebate con acierto dicha argumentación y afirma que si bien es cierto que la potestad de autoorganización corresponde a toda Administración pública y, caracterizada por su amplia discrecionalidad, permite la suscripción de un convenio interadministrativo como el que se impugna, en cualquier caso, deben respetarse las exigencias de libre concurrencia y publicidad. De manera que los Ayuntamientos podrán convenir un determinado ámbito de regulación como es «la ayuda para la prestación de un servicio, pero esta potestad de autoorganización ha de cohonestarse con el resto del ordenamiento jurídico al objeto de constatar si, efectivamente, la misma no vulnera normas imperativas» ${ }^{17}$.

Es también interesante la Sentencia del Tribunal Superior de Justicia de Castilla-La Mancha de 30 de octubre de $1998^{18}$. En ella se discute la nulidad del Convenio interadministrativo de colaboración suscrito entre dos Ayuntamientos para la prestación y gestión del servicio público de aguas potables a través de empresa municipal constituida por otra entidad local ${ }^{19}$. La Sentencia se pronuncia en términos semejantes a la anterior, declarando nulo de pleno derecho el acuerdo adoptado. Se pronuncia en los siguientes términos: «... el convenio encubre una auténtica concesión o cesión de la gestión del servicio público por un tercero distinto de la propia Administración Local titular del servicio que hasta ese momento venía prestándolo en forma directa, siendo ese tercero en este caso la empresa municipal de otra entidad local constituida para la gestión directa del servicio de abastecimiento de agua potable en su municipio. Se utiliza la figura del convenio intermunicipal, pero esta institución no es sino una cobertura meramente nominal o formal de lo que realmente ocurre: la transferencia de potestades administrativas para la gestión de un servicio público municipal a una per-

\footnotetext{
${ }^{17}$ Fundamento Jurídico Quinto, letra h).

18 Ref. RJCA 2000/2309.

${ }^{19}$ Se trata del convenio suscrito entre el Ayuntamiento de Huete y el Ayuntamiento de Alcázar de San Juan para la prestación y gestión del servicio público de aguas potables a través de la empresa municipal Aguas de Alcázar, S.A., que presta dicho servicio en este último Ayuntamiento.
} 
sona jurídica privada que actúa en el tráfico jurídico como una sociedad mercantil» ${ }^{20}$.

A pesar de que el artículo 15 de la Ley 30/1992, de 26 de noviembre, de Régimen Jurídico de las Administraciones Públicas y del Procedimiento Administrativo Común, permite que mediante la encomienda de gestión a otros órganos o Entidades de la misma o de distinta Administración para la realización de actividades de carácter material, técnico o de servicios de la competencia de los órganos administrativos o de las Entidades de Derecho público, por razones de eficacia o cuando no se posean los medios técnicos idóneos para su desempeño, realmente quien asume derechos y deberes a través del convenio interadministrativo es la empresa municipal del Ayuntamiento o entidad cesionaria, que es una empresa mercantil sometida al Derecho privado, lo que excluye la aplicación de los convenios de encomienda de gestión (art. 15.5. L. 30/1992) ${ }^{21}$.

En definitiva, este supuesto nos sitúa ante un tema más amplio que es el de la iniciativa pública local en la economía. Como es conocido, el contrapunto al artículo 38 de nuestra Constitución es el artículo 128 CE que, entre otras cosas, establece el principio de libre iniciativa pública en la economía. Concretamente, el primer inciso del apartado $2 .^{\circ}$ de este precepto determina que: «Se reconoce la iniciativa pública en la actividad económica». No vamos a entrar, desde luego, en las tesis doctrinales acerca del alcance de esta iniciativa pública, sobre la que tanto se ha escrito y a la que me remito ${ }^{22}$. Teniendo en cuenta lo anterior, en el ámbito local el

\footnotetext{
${ }^{20}$ Fundamento Jurídico Quinto.

${ }^{21}$ Dice dicho artículo que «El régimen jurídico de la encomienda de ... no será de aplicación cuando la realización de las actividades enumeradas en el apartado primero haya de recaer sobre personas físicas o jurídicas sujetas a Derecho privado, ajustándose entonces, en lo que proceda, a la legislación correspondiente de contratos del Estado, sin que puedan encomendarse a personas o Entidades de esta naturaleza actividades que, según la legislación vigente, hayan de realizarse con sujeción al Derecho administrativo».

22 Dicha doctrina la expresa con gran claridad Arana García en su obra Las Sociedades Municipales de Gestión Urbanística, Marcial Pons, Madrid, 1997, pp. 28 y ss. El prof. Arana, respecto al requisito de la presencia de interés general o utilidad pública en la actividad económica a desarrollar, afirma con rotundidad que en toda intervención pública económica pueden diferenciarse dos momentos: en primer lugar, aquél en que la Administración, como persona jurídico-pública sometida en su totalidad al Derecho administrativo, decide iniciar una actividad económica; en segundo lugar, será el ente instrumental de que se haya dotado aquélla para su actividad económica, el que, bajo un régimen jurídico privado normalmente, interviene y actúa como un sujeto más en el mercado. Pues bien, la necesaria presencia de interés público para toda actividad administrativa que exige el art. $103 \mathrm{CE}$ estará, indudablemente, presente en ese primer momento. A partir de ahí, esa intervención perderá, no absolutamente, parte de su vinculación al ente originario y creador regulándose, por tanto, por el Derecho privado (p. 34). Afirmación ésta que comparto.
} 
contenido del artículo 128.2 CE lo encontramos en el 86 de la LRBRL. Expresamente, el número $1 .^{\circ}$ de este precepto establece que «las Entidades locales, mediante expediente acreditativo de la conveniencia y oportunidad de la medida, podrán ejercer la iniciativa pública para el ejercicio de actividades económicas conforme al artículo 128.2 de la Constitución». El número $2 .^{\circ}$, interesante también a los efectos de este trabajo, determina que «cuando el ejercicio de la actividad se haga en régimen de libre concurrencia, la aprobación definitiva corresponderá al pleno de la Corporación, que determinará la forma concreta de gestión del servicio».

En el supuesto que comentamos la empresa municipal que gestiona el servicio público de abastecimiento de agua potable en el que hemos llamado municipio principal, al pretender prestar este mismo servicio en las poblaciones de los municipios vecinos, como hemos visto, transforma su naturaleza de ser empresa prestadora de un servicio público convirtiéndose en una sociedad que participa en libre concurrencia con la iniciativa privada. Ante esta situación uno de los problemas fundamentales al que debe darse solución es el de la diferente vía procedimental que la LRBRL establece para la constitución de uno y otro tipo de sociedades. Sobre todo en lo que respecta a la exigencia del expediente acreditativo de la oportunidad y concurrencia de las sociedades fruto de la libre iniciativa pública local.

Si la exigencia de dicho expediente se interpreta como un trámite esencial del procedimiento, no cabe más que concluir que ante su ausencia la constitución de esa sociedad es nula para los fines a los que pretende servir. Si, por el contrario, se entiende que se trata de un trámite no esencial, subsanable, puede tramitarse a posteriori, con el fin de justificar la conveniencia y oportunidad de la medida. 